\title{
Filling the Bose sea: symmetric quantum Hall edge states and affine characters
}

\author{
Eddy Ardonne, ${ }^{1, *}$ Rinat Kedem,${ }^{2, \dagger}$ and Michael Stone ${ }^{1, \ddagger}$ \\ ${ }^{1}$ University of Illinois, Department of Physics \\ 1110 W. Green St. \\ Urbana, IL 61801 USA \\ ${ }^{2}$ University of Illinois, Department of Mathematics \\ 1409 W. Green St. \\ Urbana, IL 61801 USA
}

\begin{abstract}
We explore the structure of the bosonic analogues of the $k$-clustered "parafermion" quantum Hall states. We show how the many-boson wave functions of $k$-clustered quantum Hall droplets appear naturally as matrix elements of ladder operators in integrable representations of the affine Lie algebra $\widehat{s u}(2)_{k}$. Using results of Feigin and Stoyanovsky, we count the dimensions of spaces of symmetric polynomials with given $k$-clustering properties and show that as the droplet size grows the partition function of its edge excitations evolves into the character of the representation. This confirms that the Hilbert space of edge states coincides with the representation space of the $\widehat{s u}(2)_{k}$ edge-current algebra. We also show that a spin-singlet, two-component $k$-clustered boson fluid is similarly related to integrable representations of $\widehat{s u}(3)$. Parafermions are not necessary for these constructions.
\end{abstract}

\footnotetext{
*Electronic address: ardonne@uiuc.edu

$\dagger$ Electronic address: rinat@uiuc.edu

${ }^{\ddagger}$ Electronic address: $\mathrm{m}-$ stone5@uiuc.edu
} 


\section{INTRODUCTION}

Clustered quantum Hall states were introduced by Read and Rezayi [1] as a natural generalization of the paired Moore-Read (or Pfaffian) state [2] that is thought to describe the FQHE phase at at $\nu=5 / 2[3-5]$. The wave-functions of these highly-correlated electronic states are constructed out correlators of parafermion operators [6] combined with a Laughlin factor that serves to cancel unwanted poles and to maintain the overall anti-symmetry under particle exchange. This construction results in the wave-functions possessing extra zeros (i.e. beyond those required by the exclusion principle) that manifest themselves when $k+1$ electron coordinates coincide. These vanishing conditions ensure that the clustered states are exact zero-energy eigenstates for a Hamiltonian with a $k$-particle repulsive interaction. As is the case with the Pfaffian state, the clustered phases host quasi-particle excitations which obey non-abelian statistics [7-9].

Read and Rezayi noted that the parafermion construction also yields $k$-clustered boson states. It is possible that such states might be realized physically in rapidly spinning pancakes of Bose fluid [10]. Even if their creation proves difficult, however, these incompressible Bose fluids are worthy of study because they are in many ways simpler than their fermionic siblings, yet they retain most of their exotic quantum properties. The simplification arises because the $k$-clustered boson wave-functions are precisely the correlators of the generating currents of the $S U(2)$, level- $k$, Wess-Zumino-Witten model - no extra Laughlin factor being required. This observation suggests that the edge-state Hilbert space of a large droplet of such an incompressible Bose fluid will coincide with the space of integrable representations of the $\widehat{s u}(2)_{k}$ affine Lie algebra. It also explains why the effective action of for the Pfaffian Bose fluid is a $S U(2)$, level-2, Chern-Simons theory [11, 12].

In order to confirm that the edge-state Hilbert space is indeed the representation space for the current algebra, it is useful to count the number of independent symmetric wavefunctions having the required $k$-particle vanishing conditions, and with a given polynomial degree. If the droplet is confined in a parabolic trap, this degree will be proportional to the energy of the corresponding edge state. The statistical partition function of the Bose droplet should then coincide with the character for a representation of $\widehat{s u}(2)_{k}[13]$. It turns out that this count was made by Feigin and Stoyanovsky [14] some years before the $k$-clustered states were introduced into physics. In this paper we will review Feigin and Stoyanovsky's 
construction of the $k$-clustered states and fill in the combinatoric details of their counting method. We will also apply a slight modification of their construction to a two-component $k$-clustered phase $[15,16]$ and show that this leads to the characters of the $\widehat{s u}(3)_{k}$ current algebra.

In section two we review the basic properties of the integrable representations of the $\widehat{s u}(2)_{k}$ current algebra. In section three, we will show how the $k$-clustered polynomials are realized as matrix elements in a subspace of these representations, and indicate how a suitable limiting procedure (corresponding to filling the "Bose sea") should allow us to construct the the entire representation space. We then confirm that this construction works by showing that the counting formula for the $k$-clustered polynomials leads to the general level- $k$ character. We end section three by stating (without proof) the results for the character of $\widehat{s u}(3)_{k}$. Details of the derivation of that result will be presented in a forthcoming paper. In section four we present a detailed derivation of the counting formula, needed to obtain the characters of $\widehat{s u}(2)_{k}$.

We wish to stress that there is little original mathematics in the present paper. With the exception of our results on $\widehat{s u}(3)_{k}$, nearly everything is to be found in [14]. That work is, however, written for mathematicians and is consequently rather inaccessible to physicists, amongst whom it deserves to be better known. We hope that our exegesis will be of use in this regard.

\section{THE $\widehat{s u}(2)_{k}$ LIE ALGEBRA}

To make our account self contained, and in order to establish our notation, we begin with a review of the well-known properties of $\widehat{s u}(2)_{k}$ and its representations.

The finite $s u(2)$ Lie algebra is generated by the operators $e \equiv J_{+} \equiv J_{1}+i J_{2}, f \equiv J_{-} \equiv$ $J_{1}-i J_{2}$, and $h \equiv 2 J_{3}$ with commutation relations

$$
[h, e]=2 e, \quad[h, f]=-2 f, \quad[e, f]=h .
$$

The symbols $e, f, h$ are the conventional notation in the mathematical literature for a Lie algebra written in the Chevalley basis. $J_{ \pm}, J_{3}$ are more common in the physics literature. Mathematicians prefer to take $2 J_{3}$, rather than $J_{3}$, as the diagonal generator, because its eigenvalues $\lambda \equiv 2 j_{3}$ will be integers. They would also refer to this algebra as $s l_{2}$, rather 
than $s u(2)$, because the " $i$ " in the ladder operators $J_{ \pm}$make it a complexification of the real $s u(2)$ algebra. We will retain the familiar physicist's name for the algebra, but use the $e, f, h$ notation so as to facilitate comparison with [14].

The infinite-dimensional $\widehat{s u}(2)_{k}$ affine Lie algebra $[17,18]$ consists of linear combinations of operators $e_{n}, f_{n}, h_{n}, n \in \mathbb{Z}$, together with the central element $\hat{k}$. In any irreducible representation $\hat{k}$ will be proportional to the identity. Because of this, we will usually omit the "hat" and simply regard $k$ as a number called the level of the representation. In all cases of interest $k$ will be a positive integer. It is also useful to adjoin a generator $\hat{d}$ that counts the "momentum" $n$. The commutation relations are then

$$
\begin{aligned}
& {\left[e_{m}, e_{n}\right]=\left[f_{m}, f_{n}\right]=0} \\
& {\left[e_{m}, \hat{k}\right]=\left[f_{m}, \hat{k}\right]=\left[h_{m}, \hat{k}\right]=0} \\
& {\left[h_{m}, e_{n}\right]=2 e_{m+n}, \quad\left[h_{n}, f_{m}\right]=-2 f_{m+n},} \\
& {\left[e_{m}, f_{n}\right]=h_{m+n}+m \hat{k} \delta_{n+m, 0}, \quad\left[h_{m}, h_{n}\right]=2 m \hat{k} \delta_{m+n, 0},} \\
& {\left[\hat{d}, e_{n}\right]=n e_{n}, \quad\left[\hat{d}, f_{n}\right]=n f_{n}, \quad\left[\hat{d}, h_{n}\right]=n h_{n} .}
\end{aligned}
$$

The operators $\hat{k}, \hat{d}$ and $h_{0}$ commute with each other and can be simultaneously diagonalized. Representations of the algebra will therefore be spanned by vectors $|m, \lambda, i\rangle$ with

$$
\hat{k}|m, \lambda, i\rangle=k|m, \lambda, i\rangle, \quad \hat{d}|m, \lambda, i\rangle=m|m, \lambda, i\rangle, \quad h_{0}|m, \lambda, i\rangle=\lambda|m, \lambda, i\rangle .
$$

The eigenvalues $k, m$ and $\lambda$ label the weights of the representation. The extra index $i$ in $|m, \lambda, i\rangle$ is necessary because the subspace with a given weight will usually have dimension greater than one. We will omit it when the weight space is one dimensional. We also omit the label $k$ because it is the same for all states in any given irreducible representation.

A highest weight state $\left|\mathbf{v}_{0}\right\rangle$ is a state that is annihilated by all $e_{n}, f_{n}$ and $h_{n}$ with $n>0$, and also by $e_{0}$. A highest weight representation is a representation containing such a state and such that all other states in the representation may be obtained by repeated application of the generators with $n \leq 0$ to this state. By adding a constant to $\hat{d}$, if necessary, we can always take the highest weight state to obey $\hat{d}\left|\mathbf{v}_{0}\right\rangle=0$. Such a state can therefore be written as $\left|\mathbf{v}_{0}\right\rangle_{k, l}=|0, l\rangle$ where $l \equiv \lambda_{\max }$ is the largest eigenvalue of $h_{0}$ in a representation of the $\left\{e_{0}, f_{0}, h_{0}\right\}$ finite $s u(2)$ sub-algebra. 

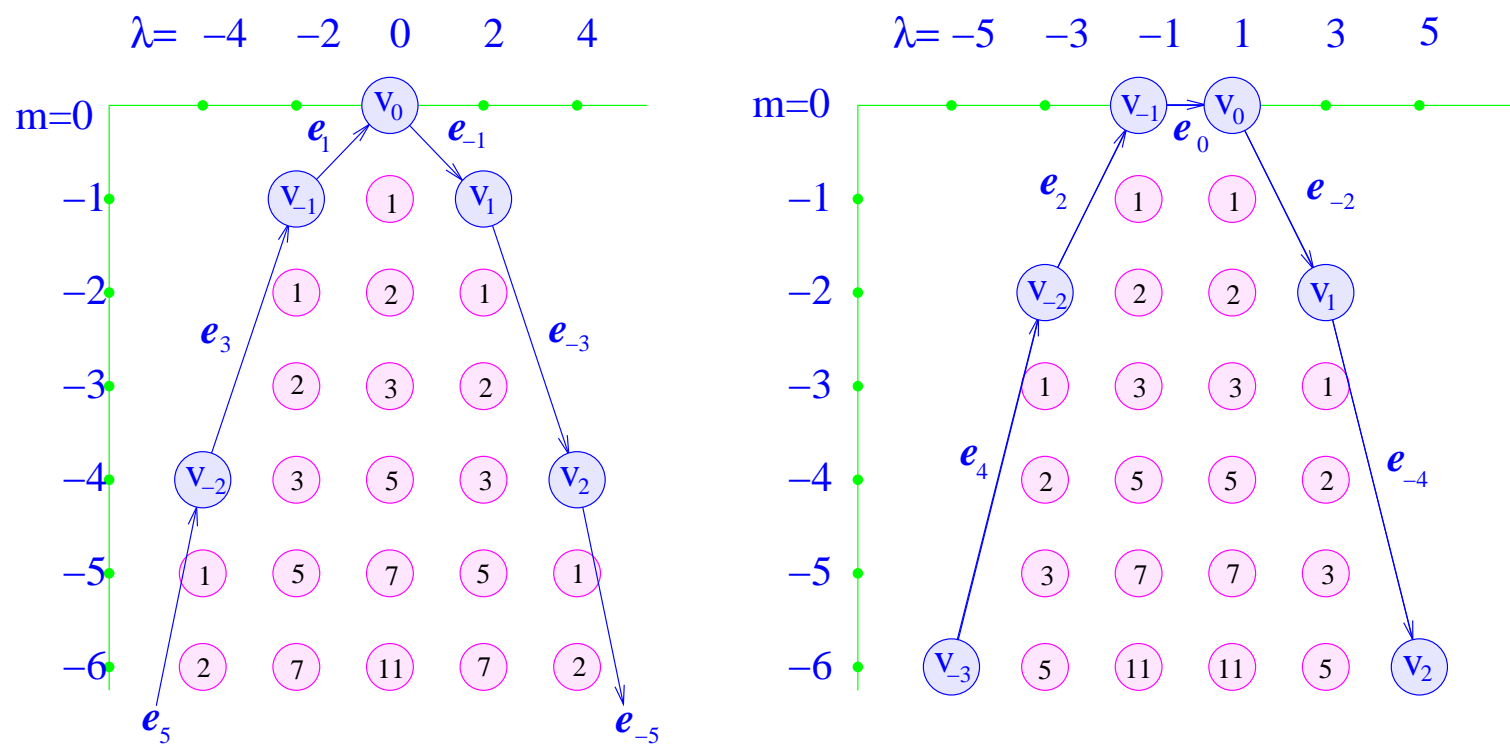

FIG. 1: Part of the weight diagrams for for the two integrable level $k=1$ representations $V_{1,0}$ and $V_{1,1}$. The number in a circle is the dimension mult $V_{k, l}(m, \lambda)$ of that weight space.

For any $n$, the generators $e_{-n}, f_{n}$ and $h_{0}-n k$ form a finite $s u(2)$ sub-algebra. A representation of the affine algebra will decompose into representations of this finite algebra. If all such sub-representations are finite dimensional, the original representation is said to be integrable. Integrable representations only occur when $k$ is a positive integer, and for such a $k$ there can be no more than $k+1$ inequivalent integrable representations. This restriction comes about because $\left|\mathbf{v}_{0}\right\rangle_{k, l}$, being killed by $f_{1}$, will be lowest weight of a representation of the $\left\{e_{-1}, f_{1}, h_{0}-k\right\}$ sub-algebra. Now $\left(h_{0}-k\right)\left|\mathbf{v}_{0}\right\rangle_{k, l}=(l-k)\left|\mathbf{v}_{0}\right\rangle_{k, l}$, and $(l-k)$, being a lowest weight in an $s u(2)$ representation, must either be zero or a negative integer. Since $l$ is either zero a positive integer, we see that $k$ must be an integer, and that the only possible values of $l$ are

$$
l=0,1,2, \ldots, k
$$

The constraints imposed by the other $\left\{e_{-n}, f_{n}, h_{0}-n k\right\}$ sub-algebras are less restrictive, and an integrable representation $V_{k, l}$ exists for each of these $l$ values. There will be exactly $(k-l)+1$ states in the $\lambda_{\max }=l$ representation of $\left\{e_{-1}, f_{1}, h_{0}-k\right\}$, and so we will have $\left(e_{-1}\right)^{k-l+1}\left|\mathbf{v}_{0}\right\rangle_{k, l}=0$. This feature can be seen in figs. 1 and 2 , where we exhibit the small $|m|$ parts of the weight diagrams for the five integrable representations at levels $k=1,2$.

The weight diagram of a representation of a finite simple Lie algebra is symmetric under the action of the Weyl group, which is generated by reflections in the planes perpendicular 

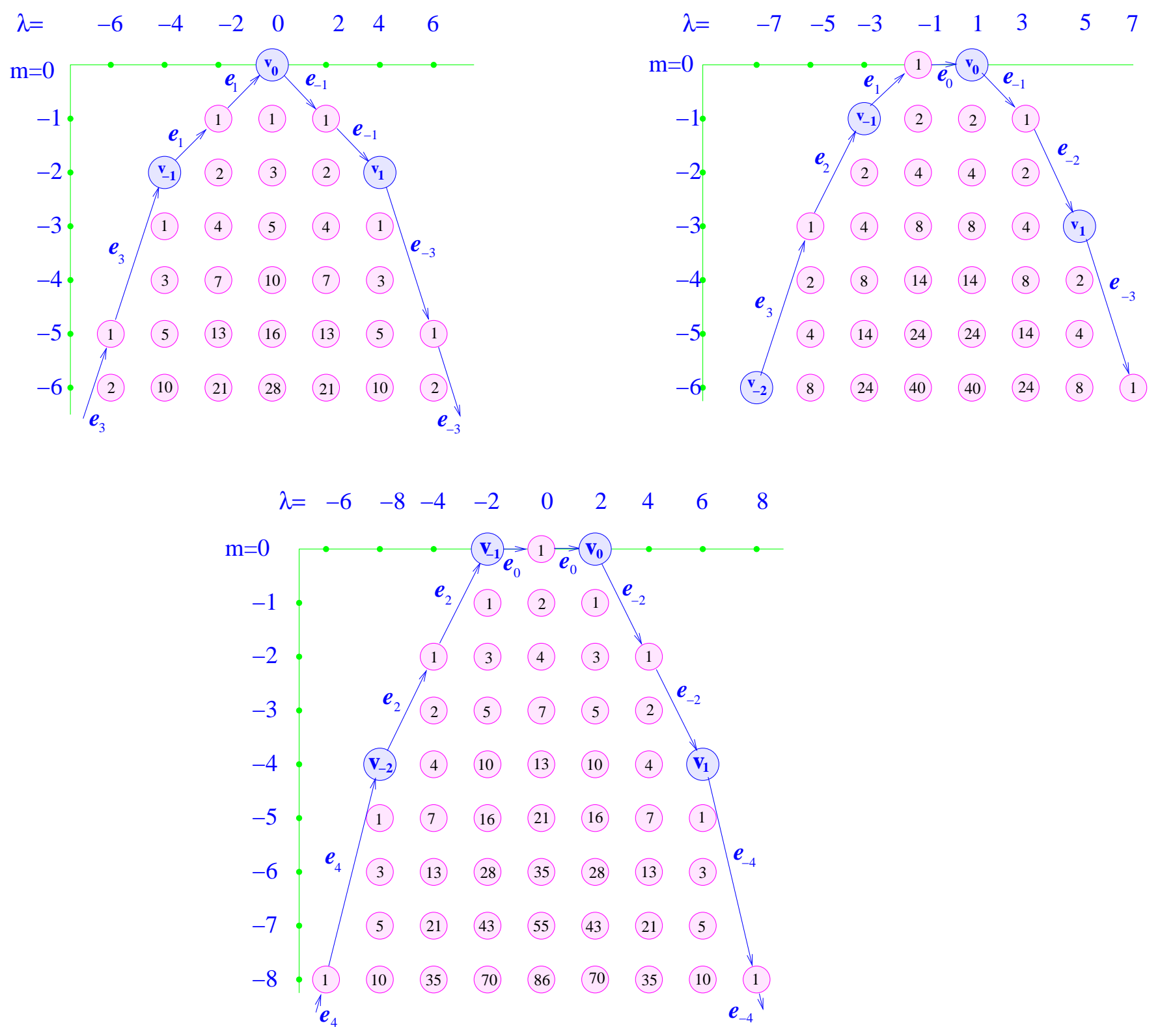

FIG. 2: Part of the weight diagram for the level $k=2$ representations $V_{2,0}, V_{2,1}$ and $V_{2,2}$.

to the root vectors. For $s u(2)$, the reflection is implemented by the action of $S=e^{i \pi J_{1}}$ on the representation. In the algebra, conjugation by $S$ takes $J_{3} \rightarrow-J_{3}$ and $J_{+} \rightarrow J_{-}$. The Weyl symmetry is an automorphism of the Lie algebra. In the affine algebra $\widehat{s u}(2)_{k}$, the weight diagram is symmetric under the action of the infinite affine Weyl group. This group is generated by the finite $S$, together with powers of a translation $T$. The action of $T$ on the algebra corresponds to conjugation by the element $g(\theta)=e^{2 \pi i h \theta}$ of the associated loop group $\tilde{L} S U(2)$. Here $\theta$ is a coordinate on the circle $S^{1}$ parameterizing the loop, and the loop $g(\theta)$ is a closed geodesic in the finite $S U(2)$ group manifold. The affine Weyl group acts on 
the weight space as

$$
\begin{aligned}
T^{n}|m, \lambda\rangle & =\left|m-\lambda n-k n^{2}, \lambda+2 k n\right\rangle, \\
S|m, \lambda\rangle & =|m,-\lambda\rangle .
\end{aligned}
$$

Weights related by this transformation will have the same multiplicities. We again see that integrability requires $k$ to be an integer. If it were not, the action of $T$ would not preserve the $\Delta \lambda=2$ spacing of the $\lambda$ eigenvalues.

In figs 1 and 2 the larger circles with labels $\mathbf{v}_{n}$ represent the orbit $\left\{\left|\mathbf{v}_{n}\right\rangle \equiv T^{n}\left|\mathbf{v}_{0}\right\rangle\right\}$ of the highest weight vector $\left|\mathbf{v}_{0}\right\rangle$ under the action of the translation part of the affine Weyl group. (We omit the subscripts on $\left|\mathbf{v}_{0}\right\rangle$ when they are unnecessary.) The $\left|\mathbf{v}_{n}\right\rangle$ are non-degenerate. The arrows indicate how the states in this orbit can be obtained from one another by the action of suitable $e_{n}$ 's.

We will also have cause to refer to the Virasoro algebra that acts on a representation $V_{k, l}$ by

$$
L_{n}=\frac{1}{2(k+2)}: \sum_{i+j=n}\left(e_{i} f_{j}+f_{i} e_{j}+\frac{1}{2} h_{i} h_{j}\right):
$$

The normal-ordering symbols ": : means that operators with positive indices $i, j$ are placed to the right of those with negative indices. Acting on $V_{k, l}$, the $L_{n}$ obey

$$
\left[L_{n}, L_{m}\right]=(n-m) L_{n+m}+\frac{c}{12} n\left(n^{2}-1\right) \delta_{n+m, 0}
$$

with central charge $c=3 k /(k+2)$. The operator $L_{0}$ acts on $V_{k, l}$ as

$$
L_{0}=\frac{j(j+1)}{(k+2)}-\hat{d}
$$

Thus $L_{0}+\hat{d}$ is the affine analogue of the quadratic Casimir operator, and takes the same value on every state in the representation $V_{k, l}$. We also have $\left[L_{-n}, e_{m}\right]=-m e_{m-n}$, together with two similar equations where $e_{m}$ is replaced by $f_{m}$ and $h_{m}$. 


\section{STRING FUNCTIONS, POLYNOMIALS AND CHARACTERS}

\section{A. String functions and characters}

The $q$-series generating function that encodes the multiplicities in the column labelled $\lambda$ of the weight diagram of $V_{k, l}$ is the string function $\sigma_{\lambda}^{k, l}(q)$. It is defined to be

$$
\sigma_{\lambda}^{k, l}(q)=\sum_{m=0}^{\infty} \text { mult }_{V_{k, l}}(m, \lambda) q^{-\left(m-m_{0}\right)} .
$$

Here $m_{0}$ is chosen to make the first power of $q$ in the sum equal to $q^{0}=1$.

Columns that are taken into each other by the action of the affine Weyl group have identical string functions. Because of this the two $k=1$ representations have only one distinct string function. For example

$$
\sigma_{0}^{1,0}(q)=1+q+2 q^{2}+3 q^{3}+5 q^{4}+7 q^{5}+11 q^{6}+\cdots=\frac{1}{(q)_{\infty}} .
$$

Here the standard notation $(q)_{n}$ means

$$
(q)_{n} \equiv \prod_{m=1}^{n}\left(1-q^{m}\right),
$$

and, in particular,

$$
(q)_{\infty} \equiv \prod_{m=1}^{\infty}\left(1-q^{m}\right) .
$$

The expression $Z_{\text {boson }} \equiv 1 /(q)_{\infty}$ is the partition function for a free chiral boson with periodic boundary conditions, but no winding numbers.

The representations $V_{2,0} \quad V_{2,2}$ contain two Weyl-inequivalent columns, and so there are two distinct string functions

$$
\begin{gathered}
\sigma_{0}^{2,0}(q)=1+q+3 q^{2}+5 q^{3}+10 q^{4}+16 q^{5}+28 q^{6}+\cdots=\frac{1}{(q)_{\infty}} \sum_{n \text { even }} \frac{q^{n^{2} / 2}}{(q)_{n}}, \\
\sigma_{2}^{2,0}(q)=1+2 q+4 q^{2}+7 q^{3}+13 q^{4}+21 q^{5}+35 q^{6}+\cdots=\frac{q^{-1 / 2}}{(q)_{\infty}} \sum_{n \text { odd }} \frac{q^{n^{2} / 2}}{(q)_{n}} .
\end{gathered}
$$

The other level-2 representation has only one distinct string function

$$
\begin{aligned}
\sigma_{1}^{2,1}(q)=1+2 q+4 q^{2}+8 q^{3}+14 q^{4}+24 q^{5}+40 q^{6}+\cdots & =\frac{1}{(q)_{\infty}} \sum_{n \text { even }} \frac{q^{n(n-1) / 2}}{(q)_{n}} \\
& =\frac{1}{(q)_{\infty}} \sum_{n \text { odd }} \frac{q^{n(n-1) / 2}}{(q)_{n}} \\
& =\frac{1}{(q)_{\infty}} \prod_{n=1}^{\infty}\left(1+q^{n}\right) .
\end{aligned}
$$


The three equalities in (14) can be extracted from the more general $q$-series [19]

$$
\sum_{n=0}^{\infty} \frac{q^{n(n-1) / 2} x^{n}}{(q)_{n}}=(1+x)(1+x q)\left(1+x q^{2}\right) \cdots
$$

The product

$$
Z_{\text {Majorana }}^{+} \equiv \prod_{n=1}^{\infty}\left(1+q^{n}\right)
$$

appearing in the last line of (14) is the partition function for an arbitrary number (i.e. chemical potential zero) of free chiral Majorana fermions with periodic boundary conditions. Combining the sum over the even and odd integers that occurs in the $l=0,2$ representations gives

$$
\sum_{n=0}^{\infty} \frac{q^{n^{2} / 2}}{(q)_{n}}=Z_{\text {Majorana }}^{-} \equiv \prod_{n=1}^{\infty}\left(1+q^{n+1 / 2}\right),
$$

which is the partition function for an arbitrary number of free chiral Majorana fermions with anti-periodic boundary conditions. The sum over even integers captures that part of the partition function with an even number of Majorana particles and the sum over the odd integers the part with an odd number of particles ${ }^{1}$.

The string functions are assembled into the characters

$$
\operatorname{ch}_{V_{k, l}}(q, x)=\sum_{m, \lambda} \operatorname{mult}_{V_{k, l}}(m, \lambda) q^{-m} x^{\lambda}
$$

Thus, for example,

$$
\begin{aligned}
\operatorname{ch}_{V_{1,0}}(q, x) & =\frac{1}{(q)_{\infty}} \sum_{n=-\infty}^{\infty} q^{n^{2}} x^{2 n} \\
\operatorname{ch}_{V_{1,1}}(q, x) & =\frac{1}{(q)_{\infty}} \sum_{n=-\infty}^{\infty} q^{n(n+1)} x^{2 n+1} .
\end{aligned}
$$

\section{B. Symmetric polynomials}

To obtain the many-boson wave functions, we begin by defining the field

$$
e(z)=\sum_{n=-\infty}^{\infty} e_{n} z^{-n-1}
$$

\footnotetext{
${ }^{1}$ The ubiquity of Majorana fermions at $k=2$ is connected with the three primary WZW fields $\phi_{m}^{j=1}$, $m=-1,0,1$, having scaling dimension $1 / 2$, and so being identifiable as linear combinations of the triplet of Majorana fermion fields that generate the $k=2$ current algebra [11].
} 
This is not quite the $e(z)$ appearing in [14], but is the customary expansion of a field of conformal dimension one. With our definition, for example,

$$
\begin{aligned}
{\left[L_{-1}, e(z)\right] } & =\partial_{z} e(z) \\
{\left[L_{0}, e(z)\right] } & =e(z)+z \partial_{z} e(z) .
\end{aligned}
$$

Because $e_{n}\left|\mathbf{v}_{0}\right\rangle=0$ for all $n \geq 0$, the equation $\left(e_{-1}\right)^{k+1-l}\left|\mathbf{v}_{0}\right\rangle=0$ can be written as

$$
\left.(e(z))^{k+1-l}\left|\mathbf{v}_{0}\right\rangle\right|_{z=0}=0
$$

Also the equation $(e(z))^{k+1}=0$ holds as an operator identity in any integrable representation of level $k$. That this is true for the $V_{k, 0}$ representation follows immediately from $(e(0))^{k+1}\left|\mathbf{v}_{0}\right\rangle=0$ by using the infinitesimal translation property of $L_{-1}$, coupled with that fact that $L_{-1}\left|\mathbf{v}_{0}\right\rangle_{k, 0}=0$. We can show, by manipulations with the primary fields of the associated WZW model, that $(e(z))^{k+1}=0$ remains true in the other integrable representations.

Now consider an integrable representation $V=V_{k, l}$, and in it the sub-space $W$ spanned by a states of the form $e_{-i_{1}} e_{-i_{2}} \ldots e_{-i_{m}}\left|\mathbf{v}_{0}\right\rangle$. We construct the matrix elements

$$
F_{\mathbf{v}}\left(z_{1}, z_{2}, \ldots, z_{p}\right) \equiv\left\langle\mathbf{v}\left|e\left(z_{1}\right) e\left(z_{2}\right) \ldots e\left(z_{p}\right)\right| \mathbf{v}_{0}\right\rangle
$$

where $|\mathbf{v}\rangle$ lies in one of the weight spaces in $V$. There are at most a finite number of products of $e_{-n}$ that can take us from $\left|\mathbf{v}_{0}\right\rangle$ to $|\mathbf{v}\rangle$, and so $F_{\mathbf{v}}\left(z_{1}, z_{2}, \ldots, z_{p}\right)$ will be a polynomial in the variables $z_{i}$. Because the $e_{n}$ commute with each other, $F_{\mathbf{v}}\left(z_{1}, z_{2}, \ldots, z_{p}\right)$ is a symmetric polynomial. If $|\mathbf{v}\rangle=|m, \lambda, i\rangle$, this polynomial will be homogeneous of degree $\operatorname{deg}\left(F_{\mathbf{v}}\right)$, with

$$
\begin{aligned}
-m & =\operatorname{deg}\left(F_{\mathbf{v}}\right)+p, \\
\lambda & =l+2 p .
\end{aligned}
$$

The equations $(e(z))^{k+1}=0$ and $\left(e_{-1}\right)^{k+1-l}\left|\mathbf{v}_{0}\right\rangle=0$ force the polynomials to possess the following properties:

i) For all $\mathbf{v}$, the polynomial $F_{\mathbf{v}}\left(z_{1}, z_{2}, \ldots, z_{p}\right)$ must vanish when more than $k$ of the $z_{i}$ coincide. (It may also vanish when $k$ or fewer points coincide.)

ii) For all $\mathbf{v}$, the polynomial $F_{\mathbf{v}}\left(z_{1}, z_{2}, \ldots, z_{p}\right)$ must vanish if $k+1-l$ of the $z_{i}$ become zero. (Again, it may also vanish when fewer than this number of $z_{i}$ gather at $z=0$.) 
The $F_{\mathbf{v}}$ thus have the $k$-cluster vanishing properties, and, in addition, have extra zeros located at the origin that correspond to the insertion there of $k-l$ quasi-holes. When multiplied by a suitable gaussian factor $\exp \left(-\Omega \sum_{i}\left|z_{i}\right|^{2} / 4\right)$ and with $z=x+i y$, they become the many-body wave functions of the two-dimensional, $k$-clustered, Bose gas phase.

There is a one-to-one correspondence between states in the sub-space $W$ and the those in the space

$$
\mathcal{F}=\bigoplus_{p=0}^{\infty} \mathcal{F}_{p},
$$

where $\mathcal{F}_{p}$ is the space of polynomials $F_{\mathbf{v}}$ in $p$ variables. To see why this is so, we need first to realize that the $F_{\mathbf{v}}$ are really labelled by the bra vector $\langle\mathbf{v}|$, which is an element of the dual space $W^{*}$. We are therefore claiming that the linearly independent $k$-clustered symmetric polynomials form a basis for $W^{*}$. If there were no dependencies between between the $e_{i}$ then $W^{*}$ would be the entire space of symmetric polynomials - this being the usual correspondence between the first- and second-quantized version of a bosonic Fock space. There are, however, linear relations between the vectors $e_{-i_{1}} e_{-i_{2}} \ldots e_{-i_{p}}\left|\mathbf{v}_{0}\right\rangle$ due to the vanishing of the Fourier components of $(e(z))^{k+1}$. Thus

$$
S_{m}=\sum_{i_{1}+i_{2}+\ldots+i_{k+1}=m} e_{i_{1}} e_{i_{2}} \ldots e_{i_{k+1}}=0
$$

where we can restrict the sum to $e_{i}$ with $i<0$. There are also relations arising from $\left(e_{-1}\right)^{k-l+1}$ being zero when acting on the vacuum. The $S_{m}$ 's, together with $\left(e_{-1}\right)^{k-l+1}$, generate an ideal $\mathcal{I}$ in the free commutative algebra $U(e)$ consisting of linear combinations of monomials in the $e_{i}, i<0$. The space $W$ can be identified with the quotient algebra $U(e) / \mathcal{I}$. In order to be uniquely defined as an element of $W^{*}$, any $\langle\mathbf{v}| \in W^{*}$ must give zero when paired with a state in the space $\mathcal{I}|\mathbf{v}\rangle_{0}$. This, however, is precisely what the vanishing properties of the polynomials enforce. The linearly independent polynomials do therefore form a basis for $W^{*}$.

We now observe that, although $W$ and $W^{*}$ are both infinite dimensional, the individual weight spaces are finite dimensional. There is therefore a one-to-one correspondence between $|m, \lambda, i\rangle \in W$ and the dual basis $\langle m, \lambda, i| \in W^{*}$. The dimensions of the space of $k$-clustered polynomials of appropriate degree, and in an appropriate number of variables, do therefore coincide with the dimensions of the weight spaces in $W$.

Not every state in $V$ can be obtained as $e_{-i_{1}} e_{-i_{2}} \ldots e_{-i_{p}}\left|\mathbf{v}_{0}\right\rangle$. Since each application of 
an $e_{-n}$ moves us one step to the right and $n$ steps down, we cannot reach any weight to the left of the central column in the weight diagram. Furthermore, we can reach weights

close to the central column via fewer distinct products $e_{-i_{1}} e_{-i_{2}} \ldots e_{-i_{p}}$ than the dimension of the weight space. Because of this paucity of paths, even if all the $e_{-i_{1}} e_{-i_{2}} \ldots e_{-i_{p}}\left|\mathbf{v}_{0}\right\rangle$ were linearly independent, we would only be able to obtain a restricted class of states in the weight space. If, however, we look at weights in columns far to the right in the weight diagram, the number of distinct products leading to a given weight grows rapidly, whilst the dimensions of the weight spaces near the head of any such column remain small. It is plausible, therefore (and we will see that it is true), that we can obtain all states in such a weight space by applying suitable products of $e_{-n}$ 's to $\left|\mathbf{v}_{0}\right\rangle$. Thus, by counting the number of symmetric polynomials with the given vanishing properties, we can obtain the early terms in the string functions. Then, by taking suitable limits, we can obtain all the terms in the string functions.

\section{Filling the sea}

To illustrate these ideas, consider the two $k=1$ representations. The general $p$-variable symmetric polynomial satisfying the $l=0$ vanishing conditions is

$$
F\left(z_{1}, z_{2}, \ldots, z_{p}\right)=S\left(z_{1}, z_{2}, \ldots, z_{p}\right) \prod_{i<j}\left(z_{i}-z_{j}\right)^{2}
$$

where $S\left(z_{1}, z_{2}, \ldots, z_{p}\right)$ is a general symmetric polynomial in $p$ variables. The number of general symmetric polynomials of degree $n$ in $p$ variables is given by the coefficient of $q^{n}$ in $1 /(q)_{p}$, and the degree of the remaining factor is $p(p-1)$. Using $-m=\operatorname{deg}(F)+p$ and $\lambda=2 p$, we find that the contribution of the $e_{-i_{1}} \ldots e_{-p}\left|\mathbf{v}_{0}\right\rangle \in W$ to the $\operatorname{ch}_{V_{1,0}}$ character is

$$
\operatorname{ch}_{W}(q, x)=\sum_{p=0}^{\infty} \frac{1}{(q)_{p}} q^{p^{2}} x^{2 p}
$$

Comparing this with the full character (19), and observing that the first $p$ terms in $1 /(q)_{p}$ and $1 /(q)_{\infty}$ coincide, we see that the first $p$ weights in each column are correctly counted.

For the case $k=1, l=1$, the general polynomial is

$$
F\left(z_{1}, z_{2}, \ldots, z_{p}\right)=S\left(z_{1}, z_{2}, \ldots, z_{p}\right) \prod_{i, j}\left(z_{i}-z_{j}\right)^{2} \prod_{i=1}^{p} z_{i}
$$


The product factors have degree $p^{2}$. The contribution of the $e_{-i_{1}} \ldots e_{-i_{p}}\left|\mathbf{v}_{0}\right\rangle$ states is therefore

$$
\operatorname{ch}_{W}(q, x)=\sum_{p=0}^{\infty} \frac{1}{(q)_{p}} q^{p(p+1)} x^{2 p+1} .
$$

Again comparison with (19) shows that the first $p$ states in each column are correctly counted.

To get the entire representation we therefore look at the states near the heads of the columns surrounding $\left|\mathbf{v}_{N}\right\rangle$. We map these weights back to the neighbourhood of $\left|\mathbf{v}_{0}\right\rangle$ by using the Weyl translation $T^{-N}$ and so find all the states near the highest-weight "vacuum". We then send $N$ to infinity, and so find all the states in an arbitrarily large neighbourhood $\left|\mathbf{v}_{0}\right\rangle$.

The state $\left|\mathbf{v}_{N}\right\rangle$ corresponds to an undeformed droplet containing $k N$ bosons. In mapping it back via the affine Weyl group to the "vacuum" state $\left|\mathbf{v}_{0}\right\rangle=T^{-N}\left|\mathbf{v}_{N}\right\rangle$ we also map the original highest-weight state to $\left|\mathbf{v}_{-N}\right\rangle$, which becomes the new no-particle state. The relation

$$
\left|\mathbf{v}_{0}\right\rangle=e_{0}^{l} e_{1}^{k-l}\left|\mathbf{v}_{-1}\right\rangle
$$

which is illustrated by the arrows in the weight diagrams, can be generalized to

$$
\left|\mathbf{v}_{0}\right\rangle=\left(e_{0}^{l} e_{1}^{k-l}\right)\left(e_{2}^{l} e_{3}^{k-l}\right) \ldots\left(e_{2 N-2}^{l} e_{2 N-1}^{k-l}\right)\left|\mathbf{v}_{-N}\right\rangle
$$

This process of adding $N k$ particles to the no-particle state corresponds to building up the droplet by filling the "Bose sea" . That we only get a full representation of the current algebra in the limit of a large droplet is familiar from the case of the $\nu=1$ Hall effect [21].

\section{Level- $k$ characters}

We now write down the $q$-series generating function that counts the general level- $k$ polynomials. Because it is rather lengthy, we defer the proof of this counting formula to the next section.

When there are $p$ variables $z_{i}$, the generating function takes the form of a sum over the partitions of $p$ into parts, each of which is no larger than $k$. These $k$-restricted partitions are most conveniently pictured as Young diagrams whose shape is described by the integers

$m_{1}, \ldots, m_{k}$. Here $m_{\alpha}$ is the number of rows containing $\alpha$ boxes. These definitions are illustrated in fig. 3 . 


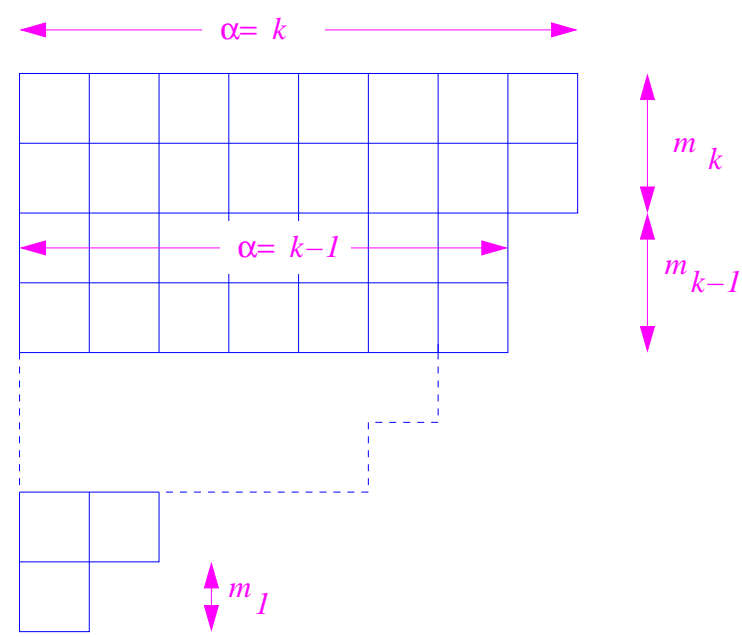

FIG. 3: Young diagram representing a $k$-restricted partition of $p$.

For the spaces $\mathcal{F}_{p}(d)$ of $k$-clustered symmetric polynomials of degree $d$ in $p$ variables, Feigin and Stoyanovsky show that [14]

$$
\sum_{d} \operatorname{mult}(p, d) q^{d}=q^{-p} \sum_{\substack{k-\text { restricted } \\ \text { partitions } \\ \text { of } p}} \frac{q^{\mathbf{m}^{t} \mathbf{M m}+\mathbf{d}^{t} \mathbf{m}}}{(q)_{m_{1}}(q)_{m_{2}} \ldots(q)_{m_{k}}}
$$

Here the $m_{\alpha}$ are the positive integers describing the partition of $p$, and so they are constrained by

$$
m_{1}+2 m_{2}+\cdots+k m_{k}=p
$$

The matrix $\mathbf{M}$ has entries $M_{\alpha \beta}=\min (\alpha, \beta)$. The vectors $\mathbf{m}$ and $\mathbf{d}$ are defined by

$$
\begin{aligned}
\mathbf{m} & =\left(m_{1}, m_{2}, \ldots, m_{k}\right)^{t}, \\
\mathbf{d} & =(0, \ldots, 0,1,2, \ldots, l)^{t},
\end{aligned}
$$

the non-zero entries in $\mathbf{d}$ beginning at $\alpha=k-l+1$.

From (33) we obtain the contribution to the character of the states in the restricted subspace $W$. We do this by setting $-m=d+p, \lambda=2 p+l$, and summing over $p$. Thus

$$
\operatorname{ch}_{W_{k, l}}(q, x)=\sum_{p=0}^{\infty} x^{2 p+l}\left\{\sum_{m_{1}+2 m_{2}+\ldots k m_{k}=p} \frac{q^{\mathbf{m}^{t} \mathbf{M m}+\mathbf{d}^{t} \mathbf{m}}}{(q)_{m_{1}}(q)_{m_{2}} \ldots(q)_{m_{k}}}\right\} .
$$


The quadratic form in the exponent can be diagonalized by setting

$$
\begin{aligned}
N_{1} & =m_{1}+m_{2}+\cdots+m_{k}, \\
N_{2} & =m_{2}+\cdots+m_{k}, \\
& \vdots \\
N_{k} & =m_{k},
\end{aligned}
$$

whence

$$
\mathbf{m}^{t} \mathbf{M m}=N_{1}^{2}+N_{2}^{2}+\cdots+N_{k}^{2} .
$$

In terms of the $N_{\alpha}$, we have

$$
\operatorname{ch}_{W_{k, l}}(q, x)=\sum_{p=0}^{\infty} x^{2 p+l}\left\{\sum_{N_{1}+\cdots+N_{k}=p} \frac{q^{N_{1}^{2}+N_{2}^{2}+\cdots N_{k}^{2}+N_{k-l+1}+N_{k-l+2}+\cdots N_{k}}}{(q)_{N_{1}-N_{2}}(q)_{N_{2}-N_{3}} \cdots(q)_{N_{k}}}\right\} .
$$

Here the $N_{\alpha}$ are restricted by $N_{1} \geq N_{2} \geq \cdots \geq N_{k} \geq 0$.

The diagonalized version of the character makes it easier to express the partial character that counts the states in the pulled-back space $T^{-N} W$. Applying $T^{-N}$ to the weights in

$$
\operatorname{ch}_{W_{k, l}}(q, x)=\sum_{\lambda, m} \operatorname{mult}_{W}(\lambda, m) x^{\lambda} q^{-m}
$$

we obtain

$$
\operatorname{ch}_{T^{-N} W_{k, l}}(q, x)=\sum_{\lambda, m} \operatorname{mult}_{W}(\lambda, m) x^{\lambda-2 k N} q^{-m-\lambda N+k N^{2}} .
$$

To apply this transformation, we observe that

$$
\lambda \equiv 2 p+l=2\left(N_{1}+N_{2}+\cdots+N_{k}\right)+l,
$$

and that there are $l$ terms in the linear part of the exponent

$$
N_{k-l+1}+N_{k-l+2}+\cdots N_{k}
$$

This suggests that we complete the squares in the quadratic form, and then change variables $N_{\alpha} \rightarrow N_{\alpha}-N$. We end up with obtain

$$
\operatorname{ch}_{T^{-N} W_{k, l}}(q, x)=\sum_{p=0}^{\infty} x^{2(p-N k)+l}\left\{\sum_{N_{1}+\cdots+N_{k}=p-N k} \frac{q^{N_{1}^{2}+N_{2}^{2}+\cdots N_{k}^{2}+N_{k-l+1}+N_{k-l+2}+\cdots N_{k}}}{(q)_{N_{1}-N_{2}}(q)_{N_{2}-N_{3}} \cdots(q)_{N_{k-1}-N_{k}}(q)_{N_{k}+N}}\right\},
$$


where $N_{1} \geq N_{2} \geq \cdots \geq N_{k} \geq-N$. It is now straightforward to take the limit $N \rightarrow \infty$ and recover the character for the complete representation

$$
\operatorname{ch}_{V_{k, l}}(q, x)=\lim _{N \rightarrow \infty} \operatorname{ch}_{T^{-N} W_{k, l}}(q, x)
$$

We find

$$
\operatorname{ch}_{V_{k, l}}(q, x)=\frac{1}{(q)_{\infty}} \sum_{N_{1} \geq N_{2} \geq \cdots \geq N_{k}} \frac{x^{2 N_{1}+2 N_{2} \cdots+2 N_{k}+l} q^{N_{1}^{2}+N_{2}^{2}+\cdots N_{k}^{2}+N_{k-l+1}+N_{k-l+2}+\cdots N_{k}}}{(q)_{N_{1}-N_{2}}(q)_{N_{2}-N_{3}} \cdots(q)_{N_{k-1}-N_{k}}}
$$

where $N_{1} \geq N_{2} \geq \cdots \geq N_{k} \in \mathbf{Z}$. This is indeed the full character of the $V_{k, l}$ representation [13], and so we have verified the assertion that all states are obtainable by application of products of $e_{-n}$ to the no-particle state $\left|\mathbf{v}_{-\infty}\right\rangle_{k, l}$.

The effect of filling the Bose sea is to make the width $k$ part of the Young diagram extend indefinitely upwards. This infinite tail corresponds to an undisturbed core of $k$ clustered bosons, whilst the $\alpha<k$ part represents a halo of partially disrupted clusters composing the edge excitations.

We close this subsection with a rather appealing form of the character (46), which will also establish the notation used in the next subsection. This form is obtained by using the inverse of the transformation (38), with the result

$$
\operatorname{ch}_{V_{k, l}}(q, x)=\frac{1}{(q)_{\infty}} \sum_{\substack{m_{1}, m_{2}, \ldots, m_{k-1} \geq 0 \\ m_{k} \in \mathbb{Z}}} x^{2 p+l} \frac{q^{\frac{1}{2} \mathbf{m}^{t} \mathbf{2} \mathbf{M m}+\mathbf{d}^{t} \mathbf{m}}}{(q)_{m_{1}}(q)_{m_{2}} \cdots(q)_{m_{k-1}}}
$$

where $p=\sum_{\alpha=1}^{k} \alpha m_{\alpha}$. In the context of the clustered quantum Hall states, the matrix 2M first appeared in [22]. After performing a final transformation

$$
m_{k}=\frac{p-\sum_{\alpha=1}^{k-1} \alpha m_{\alpha}}{k}
$$

the character takes the form

$$
\operatorname{ch}_{V_{k, l}}(q, x)=\sum_{p \in \mathbb{Z}} x^{\mathbf{A}_{1} p+l} q^{\frac{p \mathbf{A}_{1} p}{2 k}+\frac{l p}{k}}\left(\frac{1}{(q)_{\infty}} \sum_{m_{1}, \ldots, m_{k-1}}^{\prime} \frac{q^{\frac{1}{2} \mathbf{m}^{t} \mathbf{A}_{1} \mathbf{A}_{k-1}^{-1} \mathbf{m}-\left(\mathbf{A}_{k-1}^{-1} \mathbf{m}\right)_{k-l}}}{(q)_{m_{1}}(q)_{m_{2}} \ldots(q)_{m_{k-1}}}\right)
$$

where $\mathbf{A}_{k-1}^{-1}$ is the inverse Cartan matrix of $s u(k)$. Explicitly, $\left(\mathbf{A}_{k-1}^{-1}\right)_{\alpha \beta}=\min (\alpha, \beta)-\frac{\alpha \beta}{k}$ and $\left(\mathbf{A}_{k-1}\right)_{\alpha \beta}=2 \delta_{\alpha, \beta}-\delta_{|\alpha-\beta|, 1}$, where $\alpha, \beta=1, \ldots, k-1$. We used the convention that $\left(\mathbf{A}_{k-1}^{-1} \mathbf{m}\right)_{k-l}$ is zero for $l=0$ and $l=k$. 
Note that $\mathbf{A}_{1}=2$. The prime indicates the constraint $\sum_{\alpha=1}^{k-1} \alpha m_{\alpha}=p \bmod k$. The appealing feature of this form of the character is that it's explicitly expressed in terms of the string functions, which are proportional to the expression in parenthesis, c.f. eq. (18). In addition, the character is determined by $k, l$, the Cartan matrix of $s u(2)$, and the Cartan matrix of $s u(k+1)$.

\section{E. The case $\widehat{s u}(3)_{k}$.}

In [15], spin-singlet analogues of the spin-polarized Read-Rezayi states were proposed (see [16] for more details). These states have the same clustering property, namely that the wave function vanishes if any $k+1$ electron coordinates coincide. We can exploit the ladder operator strategy to construct characters of $\widehat{s u}(3)_{k}$ in a manner analogous to that of the previous sections for $\widehat{s u}(2)_{k}$. However, because of the presence of the two spin components, the situation is slightly more involved. We will be brief in this section, and refer to a forthcoming paper [20] for details.

We will here consider only the vacuum representation $V_{k}$ of $\widehat{s u}(3)_{k}$ (see [20] for arbitrary representations). In the vacuum representation, consider the subspace $W$, that is spanned by states of the form $e_{\alpha_{1},-i_{1}} e_{\alpha_{1},-i_{2}} \ldots e_{\alpha_{1},-i_{m_{1}}} f_{\alpha_{2},-i_{1}} f_{\alpha_{2},-i_{2}} \ldots f_{\alpha_{2},-i_{m_{2}}}\left|\mathbf{v}_{0}\right\rangle$. This is not the subspace $W$ exploited by Feigin and Stoyanovsky [14]. They consider the sub-space generated by the action of $e_{\alpha_{1},-i}$ and $e_{\alpha_{2},-j}$ on $\left|\mathbf{v}_{0}\right\rangle$. This may seem more natural from the viewpoint of Lie theory - after all $\alpha_{1}$ and $\alpha_{2}$ are the simple roots of the algebra - but our space has the advantage that $e_{\alpha_{1},-i}$ and $f_{\alpha_{2},-j}$ commute.

As in $\widehat{s u}(2)_{k}$ case, we construct the functions

$F_{\mathbf{v}}\left(z_{1}^{\uparrow}, z_{2}^{\uparrow}, \ldots, z_{p_{1}}^{\uparrow} ; z_{1}^{\downarrow}, z_{2}^{\downarrow}, \ldots, z_{p_{2}}^{\downarrow}\right) \equiv\left\langle\mathbf{v}\left|e_{\alpha_{1}}\left(z_{1}^{\uparrow}\right) e_{\alpha_{1}}\left(z_{2}^{\uparrow}\right) \ldots e_{\alpha_{1}}\left(z_{p_{1}}^{\uparrow}\right) f_{\alpha_{2}}\left(z_{1}^{\downarrow}\right) f_{\alpha_{2}}\left(z_{2}^{\downarrow}\right) \ldots f_{\alpha_{2}}\left(z_{p_{2}}^{\downarrow}\right)\right| \mathbf{v}_{0}\right\rangle$.

With our choice of commuting operators, these functions are symmetric polynomials in the variables $z_{i}^{\uparrow}$, and separately in the variables $z_{i}^{\downarrow}$. The polynomials $F_{\mathbf{v}}$ can therefore be interpreted as the coordinate part of wave functions of quantum Hall states, in which the spin-up (spin-down) electrons are located at $z_{i}^{\uparrow}\left(z_{j}^{\downarrow}\right)$ respectively.

In $[14]$ the objects corresponding to $F_{\mathbf{v}}$ are rational functions (which therefore can not be interpreted as wave functions for the spin-singlet quantum Hall systems), and the presence of poles complicates the derivation of the character. 


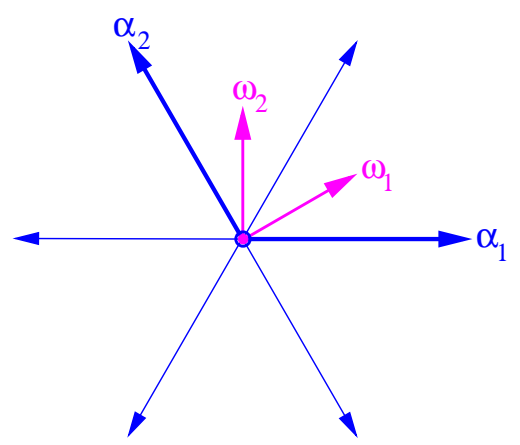

FIG. 4: The roots and weights of $s u(3)$. The vectors $\alpha_{1}$ and $\alpha_{2}$ are the simple roots, and $\omega_{1}$ and $\omega_{2}$ are the fundamental weights.

For similar reasons to those adduced the $\widehat{s u}(2)_{k}$ case, we have a set of relations $\left(e_{\alpha_{1}}(z)\right)^{k+1-a}\left(f_{\alpha_{2}}(z)\right)^{a}=0, a=0,1, \ldots, k+1$, which are valid in any integrable representation. For the special case of the vacuum representation, we have additional relations $\left(e_{\alpha_{1},-1}\right)^{k+1}\left|\mathbf{v}_{0}\right\rangle=0$ and $f_{\alpha_{2}, 0}\left|\mathbf{v}_{0}\right\rangle=0$. From these relations, it follows that $F_{\mathbf{v}}$ must be zero if any $k+1$ variables coincide (and it may vanish when fewer variables coincide).

Before we can state the character of the subspace $W$, we first have to introduce a labelling of the weight spaces. As was the case for $\widehat{s u}(2)_{k}$, the representations of $\widehat{s u}(3)_{k}$ are spanned by vectors $|\mathbf{v}\rangle=|m, \lambda, i\rangle$. In this case, however, $\lambda \equiv \lambda_{1} \omega_{1}+\lambda_{2} \omega_{2}$ is a weight of $s u(3)$. Here $\omega_{1}$ and $\omega_{2}$ are the fundamental weights (the highest weights of the 3 and $\overline{3}$ representations) and $\lambda_{1}$ and $\lambda_{2}$ are integers. For $|\mathbf{v}\rangle=|m, \lambda, i\rangle$, the polynomial $F_{\mathbf{v}}\left(z_{1}^{\uparrow}, z_{2}^{\uparrow}, \ldots, z_{p_{1}}^{\uparrow} ; z_{1}^{\downarrow}, z_{2}^{\downarrow}, \ldots, z_{p_{2}}^{\downarrow}\right)$ is of homogeneous degree $\operatorname{deg}\left(F_{\mathbf{v}}\right)$, with (compare with the analogous relations for $\widehat{s u}(2)_{k}$ in $(24))$

$$
\begin{aligned}
-m & =\operatorname{deg}\left(F_{\mathbf{v}}\right)+p_{1}+p_{2} \\
\lambda & =\left(\lambda_{1}, \lambda_{2}\right)=\left(2 p_{1}+p_{2},-\left(p_{1}+2 p_{2}\right)\right) .
\end{aligned}
$$

The character for the subspace $W$ now takes the following form

$$
\operatorname{ch}_{W_{k, 0,0}}\left(q, x_{1}, x_{2}\right)=\sum_{\substack{m_{1}, \ldots, m_{k} \geq 0 \\ n_{1}, \ldots, n_{k} \geq 0}} \frac{\left(x_{1}\right)^{2 p_{1}+p_{2}}\left(x_{2}\right)^{-\left(p_{1}+2 p_{2}\right)} q^{\frac{1}{2}\left(\mathbf{m}^{t} \mathbf{2} \mathbf{M m}+\mathbf{n}^{t} \mathbf{2} \mathbf{M n}+\mathbf{m}^{t} \mathbf{2 O n}\right)}}{\prod_{\alpha=1}^{k}(q)_{m_{\alpha}}(q)_{n_{\alpha}}}
$$

where the matrix $\mathbf{O}$ has entries $O_{\alpha \beta}=\max (0, \alpha+\beta-k), p_{1}=\sum_{i=1}^{k} i m_{i}$ and $p_{2}=\sum_{i=1}^{k} i n_{i}$. The matrix corresponding to bilinear form in (52) first appeared in the context of the nonabelian quantum Hall states in [22]. 
To find the character of the vacuum representation, we have to fill the sea by applying the appropriate Weyl translation $T_{\alpha_{1}-\alpha_{2}}^{-n}$, in a manner similar to the $\widehat{s u}(2)_{k}$ case (c.f. eq. (5)). We then take the limit $n \rightarrow \infty$. The resulting character can be written in the following form

$$
\operatorname{ch}_{V_{k, 0,0}}\left(q, x_{1}, x_{2}\right)=\frac{1}{(q)_{\infty}^{2}} \sum_{p_{1}, p_{2} \in \mathbb{Z}}\left(x_{1}\right)^{2 p_{1}+p_{2}}\left(x_{2}\right)^{-\left(p_{1}+2 p_{2}\right)} q^{\frac{1}{2 k} \mathbf{p}^{t} \overline{\mathbf{A}}_{2} \mathbf{p}} \sum_{\substack{m_{1}, \ldots, m_{k-1} \geq 0 \\ n_{1}, \ldots, n_{k-1} \geq 0}}^{\prime} \frac{q^{\frac{1}{2} \mathbf{a}^{t} \mathbf{A}_{2} \otimes \mathbf{A}_{k-1}^{-1} \mathbf{a}}}{\prod_{i=1}^{k-1}(q)_{m_{i}}(q)_{n_{i}}}
$$

where $\mathbf{a}^{t}=\left(m_{1}, \ldots, m_{k-1}, n_{1}, \ldots, n_{k-1}\right), F_{1}=\sum_{\alpha=1}^{k-1} \alpha m_{\alpha}, F_{2}=\sum_{\beta=1}^{k-1} \beta n_{\beta}$ and $\mathbf{p}^{t}=\left(p_{1}, p_{2}\right)$. $\overline{\mathbf{A}}_{2}$ is obtained from the Cartan matrix of $s u(3)$ by changing the sign of the off-diagonal elements. The prime indicates two constraints, $\sum_{\alpha=1}^{k-1} \alpha m_{\alpha}=p_{1} \bmod k$ and $\sum_{\beta=1}^{k-1} \beta n_{\beta}=$ $-p_{2} \bmod k$.

We have restricted the discussion to the vacuum representation, because the other representations involve some additional complications, which will be described in [20].

The character formula (53) for the vacuum representation of $\widehat{s u}(3)_{k}$ can be generalized to representations $V_{k, \lambda}$ with $\lambda=l w_{i}$ of any simply-laced affine Lie algebra $\widehat{g}_{k}$. To do this, we need to replace $\mathbf{A}_{2}$ by the Cartan matrix $\mathbf{C}_{r}$ of the corresponding finite Lie algebra $g$, whose rank we denote by $r$. In addition, we need to change the dependence on $x_{j}$.

In the end, the character of the vacuum representation takes the following form

$$
\operatorname{ch} V_{\widehat{g}_{k}}\left(q,\left\{x_{i}\right\}\right)=\frac{1}{(q)_{\infty}^{r}} \sum_{p_{1}, \ldots, p_{r} \in \mathbb{Z}} \prod_{i=1}^{r}\left(x_{i}\right)^{\left(\mathbf{C}_{r} \mathbf{p}\right)_{i}} q^{\frac{1}{2 k} \mathbf{p}^{t} \mathbf{C}_{r} \mathbf{p}} \sum_{\substack{m_{1}^{(1)}, \ldots, m_{k-1}^{(1)} \geq 0 \\ \vdots \\ m_{1}^{(r)}, \ldots, m_{k-1}^{(r)} \geq 0}}^{\prime} \frac{q^{\frac{1}{2} \mathbf{m}^{t} \mathbf{C}_{r} \otimes \mathbf{A}_{k-1}^{-1} \mathbf{m}}}{\prod_{j=1}^{r} \prod_{\alpha=1}^{k-1}(q)_{m_{\alpha}}^{(j)}}
$$

where $\mathbf{p}^{t}=\left(p_{1}, \ldots, p_{r}\right)$ and $\mathbf{m}^{t}=\left(m_{1}^{(1)}, \ldots, m_{k-1}^{(1)} ; \cdots ; m_{1}^{(r)}, \ldots, m_{k-1}^{(r)}\right)$, while the prime indicates the constraints $\sum_{\alpha=1}^{k-1} \alpha m_{\alpha}^{(j)}=p_{j} \bmod k$, for $j=1, \ldots, r$. The character for the basic representation of $\widehat{s u}(3)_{k}$, eq. (53), can be brought into the form of eq. (54) by changing the sign of the summation variable $p_{2}$. Note that the character formula eq. (54) appeared earlier, in references [23] and [24]. The strategy of our proof, which will appear in a future publication [20], is the same as the approach used in this paper for $\widehat{s u}(2)_{k}$ and is rather different from the combinatorical approach of [24]. 


\section{COUNTING THE POLYNOMIALS}

In this section we expand on the sketch provided in [14] and explain how to derive (33). This requires us to compute the dimensions of the spaces $\mathcal{F}_{p}(d)$ of degree- $d$, symmetric polynomials in $p$ variables that satisfy the $k$-cluster vanishing conditions and are additionally zero when $z_{1}=z_{2}=\ldots=z_{k-l+1}=0$. We will do this by introducing a filtration associated with $k$-restricted partitions of $p$ on the total space

$$
\mathcal{F}_{p}=\bigoplus_{d=0}^{\infty} \mathcal{F}_{p}(d)
$$

of such functions. Our discussion is a simplified version of that appearing in [25].

In this section we will follow the convention that partitions are labelled by greek letters such as $\lambda$ and $\mu$. The context should prevent confusion with the eigenvalues of $h_{0}$. We represent a partition $\lambda$ by a Young diagram whose shape is parameterized by positive integers $m_{\alpha}$, as described in the previous section and illustrated in figure 3. We order the partitions of $p$ lexicographically -i.e. we say that $\lambda>\mu$, if, as we read down from the top of the Young diagram and come to the first row that differs between the two partitions, the row in $\lambda$ is the longer. This is a total order relation: any two partitions $\lambda$ and $\mu$ are either identical or one is strictly greater than the other.

To define the filtration we write the variables $z_{i}$ into the boxes of the partition $\lambda$ in any order. We will relabel them so that $z_{i j}^{(\alpha)}$ is the variable in the $j$ 'th column of the $i$-th row of length $\alpha$. Given a partition we now define the evaluation map $\varphi_{\lambda}$ that acts on a function in $\mathcal{F}_{p}$ by setting the variables in each row equal to a common value $z_{i}^{(\alpha)}$. The result is a polynomial in $\sum m_{\alpha}$ variables that is symmetric under the interchange of variables with the same value of $\alpha$-i.e. deriving from rows of the same length. We claim that the image $\mathcal{H}_{\lambda}$ of $\mathcal{F}_{p}$ under the action of $\varphi_{\lambda}$ is the space of linear combinations of polynomials of the form

$$
H\left(\left\{z_{i}^{(\alpha)}\right\}\right)=h\left(\left\{z_{i}^{(\alpha)}\right\}\right) H_{\lambda}
$$

where $h\left(\left\{z_{i}^{(\alpha)}\right\}\right)$ is any function symmetric under the interchange of variables with the same value of $\alpha$, and

$$
H_{\lambda}=\prod_{\alpha=k-l+1}^{k} \prod_{i=1}^{m_{\alpha}}\left(z_{i}^{(\alpha)}\right)^{\alpha-k+l} \prod_{(\alpha, i)>\left(\alpha^{\prime}, i^{\prime}\right)}\left(z_{i}^{(\alpha)}-z_{i^{\prime}}^{\left(\alpha^{\prime}\right)}\right)^{2 M_{\alpha \alpha^{\prime}}}
$$


Here $M_{\alpha \beta}=\min (\alpha, \beta)$, and we are thinking of the row index $i$ increasing downward as is customary when writing matrices, so - perhaps perversely - we order the indices so that $(\alpha, i)>\left(\alpha^{\prime}, i^{\prime}\right)$ if $\alpha>\alpha^{\prime}$, or, if $\alpha=\alpha^{\prime}$, then $i<i^{\prime}$. Now set

$$
\Gamma_{\lambda}=\bigcap_{\mu>\lambda} \operatorname{ker} \varphi_{\lambda}, \quad \Gamma_{\lambda}^{\prime}=\bigcap_{\mu \geq \lambda} \operatorname{ker} \varphi_{\lambda}
$$

Thus $\Gamma_{\lambda}$ is the space of functions that annihilated by every evaluation map for $\mu>\lambda$. Clearly $\Gamma_{\lambda} \subset \Gamma_{\mu}$ if $\lambda>\mu$. This nested set of subspaces of $\mathcal{F}$ is the filtration that we require.

Observe that $\Gamma_{\lambda}^{\prime} \subset \Gamma_{\lambda}$, and $\Gamma_{\left(1^{p}\right)}^{\prime}=0$ because $\varphi_{\left(1^{p}\right)}$ conflates no variables, and is therefore an isomorphism. We can therefore define the graded space

$$
\operatorname{Gr} \Gamma=\bigoplus_{\lambda} \operatorname{Gr}_{\lambda} \Gamma
$$

where $\operatorname{Gr}_{\lambda} \Gamma=\Gamma_{\lambda} / \Gamma_{\lambda}^{\prime}$ and the sum is over partitions of $p$. Our strategy is to prove that

$$
\varphi_{\lambda}: \operatorname{Gr}_{\lambda} \Gamma \rightarrow \mathcal{H}_{\lambda}
$$

is an isomorphism of graded vector spaces. We must therefore show that this map is well defined, and that it is both injective and surjective. Because of this isomorphism, we can use the polynomials in $\mathcal{H}_{\lambda}$ rather than the polynomials in $\mathcal{F}_{p}$ to calculate the character, which makes the problem tractable.

We begin by showing that the map is injective and well-defined. To do this first observe that the image of $\Gamma_{\lambda}^{\prime}$ under the action $\varphi_{\lambda}$ is automatically zero as a consequence of the definition of $\Gamma_{\lambda}^{\prime}$. There is therefore no problem in defining the action of $\varphi_{\lambda}$ on the quotient space $\operatorname{Gr}_{\lambda} \Gamma=\Gamma_{\lambda} / \Gamma_{\lambda}^{\prime}$. Furthermore, by definition, the difference of any two functions in $\Gamma_{\lambda}$ that map down to the same function in $\mathcal{H}_{\lambda}$ lies in $\Gamma_{\lambda}^{\prime}$. The map is therefore injective.

To complete the demonstration that the map is well-defined, we must show our characterization of the space $\mathcal{H}_{\lambda}$ is correct in that its elements are indeed of the form claimed in (56) and (57). To show that $f \in \mathcal{H}_{\lambda}$ implies that $\varphi_{\lambda}(f)$ has a zero of at least $2 \min (\alpha, \beta)$ when $z_{i}^{(\alpha)}=z_{i^{\prime}}^{(\beta)}$, it is sufficient to consider the dependence of $f$ on the two set of variables $\left\{z_{i j}^{(\alpha)}\right\}_{j=1}^{\alpha}$ and $\left\{z_{i^{\prime} j}^{(\beta)}\right\}_{j=1}^{\beta}$, with $\alpha \geq \beta$. We can carry out the evaluation map in two steps: $\varphi_{\lambda}=\varphi^{1} \circ \varphi^{2}$. Here $\varphi^{1}$ consists of conflating all sets of variables except the $\left\{z_{i^{\prime} j}^{(\beta)}\right\}_{j=1}^{\beta}$, and $\varphi^{2}$ consists of setting $z_{i^{\prime} 1}^{(\beta)}=\cdots=z_{i^{\prime} \beta}^{(\beta)}=z_{i^{\prime}}^{(\beta)}$. Let

$$
f_{1}\left(z_{i}^{(\alpha)} ; z_{i^{\prime} 1}^{(\beta)}, \ldots, z_{i^{\prime} \beta}^{(\beta)}\right)=\varphi^{1}\left[f\left(\left\{z_{i}\right\}\right)\right]
$$


Now, by definition $f\left(z_{i}\right)$ is annihilated by all $\varphi_{\mu}$ with $\mu>\lambda$. Therefore

$$
\left.f_{1}\left(z_{i}^{(\alpha)} ; z_{i^{\prime} 1}^{(\beta)}, \ldots, z_{i^{\prime} \beta}^{(\beta)}\right)\right|_{z_{i^{\prime} j}^{(\beta)}=z_{i}^{(\alpha)}}=0 \quad j=1, \ldots, \beta
$$

because this corresponds to an evaluation at some partition larger than $\lambda$. Therefore,

$$
f_{1}\left(z_{i}^{(\alpha)} ; z_{i^{\prime} 1}^{(\beta)}, \ldots, z_{i^{\prime} \beta}^{(\beta)}\right)=\prod_{j=1}^{\beta}\left(z_{i}^{(\alpha)}-z_{i^{\prime} j}^{(\beta)}\right) \tilde{f}_{1}\left(z_{i}^{(\alpha)} ; z_{i^{\prime} 1}^{(\beta)}, \ldots, z_{i^{\prime} \beta}^{(\beta)}\right) .
$$

Now $f_{1}\left(z_{i}^{(\alpha)} ; z_{i^{\prime} 1}^{(\beta)}, \ldots, z_{i^{\prime} \beta}^{(\beta)}\right)$ was obtained from a symmetric function, and so, for each $j$,

$$
\left.\frac{\partial f_{1}}{\partial z_{i}^{(\alpha)}}\right|_{z_{i^{\prime} j}^{(\beta)}=z_{i}^{(\alpha)}}=\left.\alpha \frac{\partial f_{1}}{\partial z_{i^{\prime} j}^{(\beta)}}\right|_{z_{i^{\prime} j}^{(\beta)}=z_{i}^{(\alpha)}} .
$$

However (63) tells us that, again for each $j$,

$$
\left.\frac{\partial f_{1}}{\partial z_{i}^{(\alpha)}}\right|_{z_{i^{\prime} j}^{(\beta)}=z_{i}^{(\alpha)}}=-\left.\frac{\partial f_{1}}{\partial z_{i^{\prime} j}^{(\beta)}}\right|_{z_{i^{\prime} j}^{(\beta)}=z_{i}^{(\alpha)}}=\left.\prod_{j^{\prime}=1}^{\beta}\left(z_{i}^{(\alpha)}-z_{i^{\prime} j^{\prime}}^{(\beta)}\right) \tilde{f}_{1}\right|_{z_{i^{\prime} j}^{(\beta)}=z_{i}^{(\alpha)}},
$$

the prime on the product meaning that the term with $j^{\prime}=j$ is to be omitted. The only way to reconcile (64) with (65) is for $\left.\tilde{f}_{1}\right|_{z_{i^{\prime} j}^{(\beta)}=z_{i}^{(\alpha)}}$ to be zero. Thus the zero at $z_{i^{\prime} j}^{(\beta)}=z_{i}^{(\alpha)}$ is at least a double zero:

$$
f_{1}\left(z_{i}^{(\alpha)} ; z_{i^{\prime} 1}^{(\beta)}, \ldots, z_{i^{\prime} \beta}^{(\beta)}\right)=\prod_{j=1}^{\beta}\left(z_{i}^{(\alpha)}-z_{i^{\prime} j}^{(\beta)}\right)^{2} \tilde{f}_{2}\left(z_{i}^{(\alpha)} ; z_{i^{\prime} 1}^{(\beta)}, \ldots, z_{i^{\prime} \beta}^{(\beta)}\right) .
$$

We now evaluate the right-hand-side of $(66)$ at $z_{i^{\prime} 1}^{(\beta)}=\ldots=z_{i^{\prime} \beta}^{(\beta)}=z_{i^{\prime}}^{(\beta)}$ and, recalling the condition that $\alpha \geq \beta$, we have

$$
\varphi_{\lambda}\left[f\left(\left\{z_{i}\right\}\right)\right]=\left(z_{i}^{(\alpha)}-z_{i^{\prime}}^{(\beta)}\right)^{2 \min (\alpha, \beta)} \tilde{f} .
$$

Now we must establish that the image of $\varphi_{\lambda}$ acting on $f \in \mathcal{F}$ has a zero of order at least $\max (0, \alpha-k+l)$ whenever $z_{i}^{(\alpha)}=0$. To see that this is so, consider the dependence of $f$ on the variables $z_{1}, \ldots z_{\alpha}$, with $\alpha>k-l$, which are set equal to each other under the mapping $\varphi_{\lambda}$. We carry our the map by setting the variables equal to zero consecutively. The function

$$
g\left(z_{k-l+1}, \ldots, z_{\alpha} ; \ldots\right)=\left.f\right|_{z_{1}=\ldots=z_{k-l}=0},
$$

has a factor $\prod_{i=k-l+1}^{\alpha} z_{i}$ because it is zero if any of the remaining variables are set to equal to zero. Therefore if any of the $z_{i}^{\alpha}$ in the evaluation mapping are set to zero, the image has a zero of at least degree $\alpha-k+l$. 


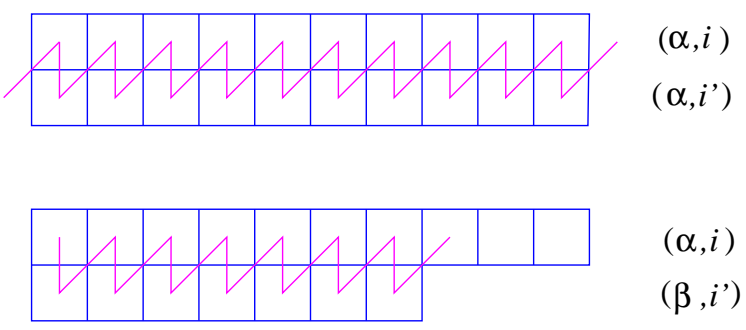

FIG. 5: The pattern on pairwise products appearing in equation (70). The top figure is for the case $\alpha=\beta$ and the second for $\alpha>\beta$. Each line joining the centers of a box corresponds to a factor $\left(z_{i}-z_{j}\right)$

The last, and hardest, task is to establish surjectivity. In other words, to show that there is at least one pre-image function in $\mathcal{F}_{p}$ for every function of the form $(56,57)$ that we claim constitutes the image $\mathcal{H}_{\lambda}$.

Let $\lambda$ be a fixed partition and consider a function $F\left(\left\{z_{i}\right\}\right)$ of the form

$$
F\left(\left\{z_{i}\right\}\right)=\operatorname{Sym} f\left(\left\{z_{i}\right\}\right)
$$

where the symmetrization is over the variables $z_{i}$ and

$$
f\left(\left\{z_{i}\right\}\right)=\tilde{h}\left(\left\{z_{i}\right\}\right) \prod_{(\alpha, i)>\left(\beta, i^{\prime}\right)}\left(z_{i j}^{(\alpha)}-z_{i^{\prime} j}^{(\beta)}\right)\left(z_{i, j+1}^{(\alpha)}-z_{i^{\prime} j}^{(\beta)}\right) \prod_{\alpha>k-l i, j>k-l} \prod_{i j} z_{i j}^{(\alpha)},
$$

where $\tilde{h}$ is a polynomial, and $z_{i, \alpha+1}^{(\alpha)} \equiv z_{i, 1}^{(\alpha)}$. (The pattern of pairwise products is illustrated in figure 5.) We claim that the function $F$ is an element of $\Gamma_{\lambda}$.

We first observe that if any $k+1$ of the $z_{i}$ are set equal, the factor $\prod\left(z_{i j}^{(\alpha)}-z_{i^{\prime} j}^{(\beta)}\right)$ is zero: Consider the Young diagram corresponding to $\lambda$. There are most $k$ columns. Since we are equating $k+1$ variables, at least two of them are forced to be in the same column and so give to a zero factor. It is easy to check that $F$ satisfies the vanishing conditions $z_{1}=\ldots=z_{k-l+1}=0$. It is therefore an element of $\mathcal{F}_{p}$.

We next show that $F$ is in the kernel of $\varphi_{\lambda}$ for any $\mu>\lambda$ : We arrange the variables $z_{i j}^{(\alpha)}$ in the boxes of the Young diagram corresponding to $\mu$ and recall that variables in the same row are set equal to each other under the evaluation map. Thus no two variables from the same column in $\lambda$ may appear in the same row in $\mu$ if the prefactor $\prod\left(z_{i j}^{(\alpha)}-z_{i^{\prime} j}^{(\beta)}\right)$ is to be non-zero. Since $\mu>\lambda$, this is impossible: Let the lengths of the rows in the partitions be denoted by $\lambda_{i}$ and $\mu_{i}$, with the index $i$ increasing downwards. Suppose that $\mu_{i}=\lambda_{i}$ for $i=1, \ldots, j$ and $\mu_{j}>\lambda_{j}$. All the variables from the rows $\lambda_{i}=k$ must appear in the rows 
of $\mu$ with the same length, similarly for rows of length $k-1$, and so on. But in row $j$ of $\mu$ there can be at most $\lambda_{j}$ variables from row $j$ of $\lambda$, and at least one other variable must come from row $j^{\prime}$ with $j^{\prime}>j$. But $\lambda_{j^{\prime}} \leq \lambda_{j}$, and hence this variable belongs to the same column of $\lambda$ as some variable in row $j$. Therefore under the evaluation mapping the image of $F$ is zero.

Now we turn to the factor $\tilde{h}$ which is to give rise to the symmetric function $h$ in (56). We can take as a basis for such functions the symmetrized monomials

$$
h=\prod_{\alpha} \sum_{\sigma \in S_{m_{\alpha}}} \sigma\left(\prod\left(z_{i}^{(\alpha)}\right)^{\lambda_{i}^{(\alpha)}}\right)
$$

Here $\lambda^{(\alpha)}$ is a partition with no more that $m_{\alpha}$ rows, these rows being of length $\lambda_{i}^{(\alpha)}$. The symmetrization is over each set of variables $\left(z_{1}^{(\alpha)}, \ldots z_{m_{\alpha}}^{(\alpha)}\right)$ for fixed $\alpha$. Let

$$
\tilde{h}\left(\left\{z_{i}\right\}\right)=\prod_{\alpha=1}^{k}\left[\prod_{i=1}^{m_{\alpha}}\left(z_{i 1}^{(\alpha)}\right)^{\lambda_{i}^{(\alpha)}}\right],
$$

be the function appearing in (70). We claim that $\varphi_{\lambda}(F)$ is a scalar multiple of the function $H$ in (56) with $h$ as in (71).

To see that this claim is correct consider the sum over the symmetric group $S_{p}$

$$
\sum_{\sigma \in S_{p}} f\left(\sigma\left(\left\{z_{i}\right\}\right)\right)
$$

Suppose that for some $\sigma$, we have $\sigma\left(z_{a b}^{(\beta)}\right)=z_{c d}^{(\gamma)}$ with $(\gamma, c)<(\beta, a)$, and that $(\beta, a)$ is the largest row for which this true. This means that all rows above $(\beta, a)$ undergo only a permutation within the row. Suppose that the prefactor

$$
\varphi_{\lambda} \circ \sigma\left(\prod_{(\alpha, i)>\left(\alpha^{\prime}, i^{\prime}\right)}\left(z_{i j}^{(\alpha)}-z_{i^{\prime} j}^{\left(\alpha^{\prime}\right)}\right)\left(z_{i, j+1}^{(\alpha)}-z_{i^{\prime} j}^{\left(\alpha^{\prime}\right)}\right)\right)
$$

is to be non-zero. Then $z_{c d}^{(\gamma)}$ cannot be in a column directly below or to the left of the permutation image of any other element from row $(\beta, a)$ - but this means that at least one other element from row $(\beta, a)$ should be mapped to a row below $(\beta, a)$. If it is mapped to the $(\gamma, c)$ it can appear in any column other than $d$. If it mapped to any other row, it can appear in any other column than $d$ and an adjacent column (to the right or left depending on whether it is above or below $(\gamma, c)$.) Now we repeat this argument for this new element, concluding that at least one more element of row $(\beta, a)$ is mapped to a lower row, and so 
forth, until eventually we find that all elements are permuted to a row below $(\beta, a)$. If the elements are permuted to the same row, they can be placed adjacent columns. Elements which are permuted to different rows can not be placed in adjacent columns, this being due to the factor linking adjacent columns in the prefactor. There are at most $\beta$ columns in $\lambda$ in rows below $(\beta, a)$, and hence the elements must all appear in the same row, which is therefore of length $\beta$. Thus all the variables in rows of length $\beta$ are mapped to another row of length $\beta$, for the same reason. As a result, he only permutations which give a non-zero contribution to $\varphi_{\lambda} \circ \sigma$ are those that which permute variables within each row, or those that permute rows of equal length. Under the evaluation map, the former contribute equal terms to the sum, while row interchanges correspond to the symmetrization over $z_{i}^{(\alpha)}$ in $(71)$.

We have now completed the proof that the map $\varphi_{\lambda}: \operatorname{Gr}_{\lambda} \Gamma \rightarrow \mathcal{H}_{\lambda}$ is an isomorphism. This map is clearly degree preserving, and so it only remains to count the number of polynomials in each space $H_{\lambda}$.

Referring back to (57) we see that the minimal degree of a polynomial associated with a partition $l$ receives a contribution from the second product of $2 m_{\alpha} m_{\beta} \min (\alpha, \beta)$ for each pair $\alpha>\beta$, and $\alpha m_{\alpha}\left(m_{\alpha}-1\right)$ for each $\alpha$. The first product makes a contribution $\sum_{\alpha} m_{\alpha} \min (0, \alpha-k+l)$. The total minimal polynomial degree is therefore

$$
\sum_{\alpha \beta} m_{\alpha} M_{\alpha \beta} m_{\beta}-\sum_{\alpha} \alpha m_{\alpha}+\sum_{\alpha} m_{\alpha} \min (0, \alpha-k+l)
$$

Using $\sum_{\alpha} \alpha m_{\alpha}=p$, the total number of variables, this becomes

$$
\mathbf{m}^{t} \mathbf{M m}+\mathbf{d}^{t} \mathbf{m}-p
$$

where $\mathbf{m}=\left(m_{1}, \ldots m_{k}\right)^{t}$ and $\mathbf{d}$ has entries $d_{i}=\min (0, \alpha-k+l)$. The generating functions for the number of symmetric polynomials $h\left(\left\{z_{i}^{(\alpha)}\right\}\right)$ of degree $d$ are $\left(\prod_{\alpha}(q)_{m_{\alpha}}\right)^{-1}$. Putting the parts together, we therefore have

$$
\sum \operatorname{mult}(p, d) q^{d}=q^{-p} \sum_{\substack{k-\text { restricted } \\ \text { partitions } \\ \text { of } p}} \frac{q^{\mathbf{m}^{t} \mathbf{M m}+\mathbf{d}^{t} \mathbf{m}}}{(q)_{m_{1}}(q)_{m_{2}} \ldots(q)_{m_{k}}}
$$

as claimed in (33). 


\section{CONCLUSION}

We have shown that a planar droplet of bosons in a strongly correlated Read-Rezayi $k$-clustered phase [1] will have low energy excitations that can be identified with states in

representations of the $\widehat{s u}(2)_{k}$ current algebra. This simple picture applies provided that the energies of these excited states are sufficiently low that only the edge of the droplet is perturbed, a central core being left intact. This picture provides a physical interpretation for the observation [14] that the space of $k$-clustered symmetric polynomials in an arbitrary number of variables is in one-to-one correspondence with states in the integrable representations of the Lie algebra $\widehat{s u}(2)_{k}$. We also presented a generalization of these results to a two component spin-singlet system, whose excitations are classified by the $\widehat{s u}(3)_{k}$ algebra.

\section{ACKNOWLEDGMENTS}

This work was supported by the National Science Foundation under grant NSF-DMR-0132990. MS and EA thank Eduardo Fradkin for many useful conversations, and RK thanks Boris Feigin and Tetsuji Miwa for discussions about counting methods.

[1] N. Read, E. Rezayi, Beyond Paired Quantum Hall States: parafermions and incompressible states in the first Landau Level, Phys. Rev. B 59 (1999) 8084.

[2] G. Moore, N. Read, Nonabelions in the fractional quantum Hall effect, Nucl. Phys. B 360 (1991) 362.

[3] M. Greiter, X.-G. Wen, F. Wilczek, Paired Hall states, Nucl. Phys. B 374 (1992) 567.

[4] R. Morf, Transition from quantum Hall to compressible states in the second Landau level: new light on the $\nu=5 / 2$ enigma, Phys. Rev. Lett. 80 (1998) 1505.

[5] N. Read, D. Green, Paired States of fermions in two-dimensions with breaking of parity and time reversal symmetries, and the fractional quantum Hall effect, Phys. Rev. B 61, 10267 (2000).

[6] A. B. Zamolodchikov, V. A. Fateev, Nonlocal (parafermion) currents in two-dimensional conformal field theory and self-dual critical points in $Z_{n}$ symmetric statistical systems, Sov. Phys. JETP 62 (1985) 215. 
[7] N. Read, E. Rezayi, Quasi-holes and fermionic zero modes of paired fractional quantum Hall states: the mechanism for non-abelian statistics, Phys. Rev. B54 (1996) 16864.

[8] C. Nayak, F. Wilczek, 2n-quasihole states realize $2^{n-1}$-dimensional spinor braiding statistics in paired quantum Hall states, Nucl. Phys. B 479 (1996) 529.

[9] J.K. Slingerland, F.A. Bais, Quantum groups and non-abelian braiding in quantum Hall systems, Nucl. Phys. B 612 (2001) 229.

[10] N.R. Cooper, N.K. Wilkin, J.M.F. Gunn, Quantum phases of vortices in rotating BoseEinstein condensates, Phys. Rev. Lett. 87 (2001) 120405.

[11] E. Fradkin, C. Nayak, A. M. Tsvelik, F. Wilczek, A Chern-Simons effective field theory for the Pfaffian quantum Hall state, Nucl. Phys. B. B516 (1998), 704.

[12] E. Fradkin, C. Nayak, K. Schoutens, Landau-Ginzburg theories of non-Abelian quantum Hall states, Nucl. Phys. B. B546 (1999), 711.

[13] J. Lepowsky, M. Primc, Structure for Standard modules of the affine Lie algebra $A_{1}^{(1)}$, Contemporary Mathematics 46 (AMS, Providence RI, 1985).

[14] A. V. Stoyanovsky, B. L. Feigin, Functional models for representations of current algebras and semi-infinite Schubert cells, Functional Analysis and its applications, 28 (1994) 55-72.

[15] E. Ardonne, K. Schoutens, New class of non-abelian spin-singlet quantum Hall states, Phys. Rev. Lett. 82 (1999) 5096.

[16] E. Ardonne, N. Read, E. Rezayi, K. Schoutens, Non-abelian spin-singlet quantum Hall states: wave functions and quasihole state counting, Nucl. Phys. B 607 (2001) 549.

[17] V. Kac, Infinite dimensional Lie algebras, (Cambridge University Press, Cambridge, England 1983).

[18] For a physicist's account, see: P. Di Franco, P. Mathieu, D. Sénéchal, Conformal Field Theory, (Springer, New York 1997), Chapter 14.

[19] See, for example: G. E. Andrews, The Theory of Partitions. (Cambridge University Press, Cambridge, England 1998)

[20] E. Ardonne, R. Kedem, M. Stone, in preparation.

[21] M. Stone, Schur functions, chiral bosons, and the quantum-Hall-effect edge states, Phys. Rev. B42 (1990) 8399.

[22] E. Ardonne, P. Bouwknegt, K. Schoutens, Non-abelian quantum Hall states - Exclusion statistics, K-matrices and duality, J. Stat. Phys. 102 (2001) 421. 
[23] A.N. Kirillov, Dilogarithm identities, Prog. Theor. Phys. Suppl. 118 (1995) 61.

[24] G. Georgiev, Combinatorial construction of modules for infinite-dimensional Lie algebras, II. Parafermionic space, q-alg/9504024 (1995).

[25] B. Feigin, M. Jimbo, R. Kedem, S. Loktev, T. Miwa, Spaces of coinvariants and fusion product II. $\widehat{s l}_{2}$ character formulas in terms of Kostka polynomials, Journal of Algebra 279 (2004), 147. 